\title{
Glide Slope Control Authority for Parafoil Canopies with Variable Incidence Angle
}

\author{
Michael Ward,,- Alek Gavrilovski,,$*$ and Mark Costello $¥$ \\ Georgia Institute of Technology, Atlanta, Georgia 30332
}

DOI: $10.2514 / 1 . C 032029$

\begin{abstract}
The addition of glide slope control to guided airdrop systems has the potential to provide dramatic improvements in landing accuracy. Dynamic rigging incidence control has been demonstrated in flight tests and shown to provide effective glide slope control. The current work presents flight-test results exploring the range of glide slope control that can be achieved with high-performance parafoil canopies of two different aspect ratios. The low- and mediumaspect-ratio canopies have peak glide ratios of 4.4 and 4.9, respectively. By varying the incidence angle in flight, the glide slope can be reduced from these peak values to a glide slope near two for both canopies. Flight tests investigating the relationship between symmetric brake and incidence control demonstrate that symmetric braking produces effective airspeed control with little effect on glide slope, the incidence angle produces significant effects on both glide slope and airspeed, the rapid variation of the incidence angle in flight can lead to significant long period oscillations in both glide slope and airspeed, and the coupled effect of the control mechanisms of incidence angle and symmetric brake can produce dramatic changes in glide slope in any wind condition.
\end{abstract}

$\begin{array}{ll} & \text { Nomenclature } \\ c & =\text { chord } \\ \mathrm{GS} & =\text { glide slope } \\ V_{A} & =\text { airspeed } \\ V_{w} & =\text { wind speed } \\ \dot{z} & =\text { descent rate } \\ \alpha & =\text { angle of attack } \\ \Gamma & =\text { incidence angle } \\ \gamma & =\text { flight-path angle } \\ \delta_{B} & =\text { symmetric brake deflection } \\ \delta L & =\text { symmetric brake deflection } \\ \delta R & =\text { symmetric brake deflection } \\ \chi & =\text { azimuth angle } \\ \psi & =\text { heading angle } \\ \omega & =\text { turn rate }\end{array}$

\section{Introduction}

A ERIAL payload delivery has historically been performed with unguided, ballistic parachutes. The use of a gliding canopy such as the parafoil offers the possibility for dramatic improvements in the accuracy of payload delivery. These systems are dropped from an aircraft at altitude and guided autonomously to a desired landing location using feedback from the Global Positioning System (GPS) as input to the guidance and control computations. Deflection of the trailing edge is typically the only method used for control. Asymmetric deflection of the trailing edge provides effective lateral control, and symmetric deflection provides effective speed control with little to no effect on the glide slope. A large number of autonomous guided airdrop systems have been developed using this basic control mechanism [1-10]. Without an effective glide slope control mechanism, these systems are forced to rely on carefully

Presented as Paper 2011-2620 at the 21st AIAA Aerodynamic Decelerator Systems Technology Conference and Seminar, Dublin, Ireland, 23-26 May 2011; received 2 July 2012; revision received 20 October 2012; accepted for publication 13 November 2012; published online 23 August 2013. Copyright (C) 2013 by Michael Ward. Published by the American Institute of Aeronautics and Astronautics, Inc., with permission. Copies of this paper may be made for personal or internal use, on condition that the copier pay the $\$ 10.00$ per-copy fee to the Copyright Clearance Center, Inc., 222 Rosewood Drive, Danvers, MA 01923; include the code 1542-3868/13 and \$10.00 in correspondence with the CCC.

* Graduate Research Assistant, School of Aerospace Engineering. Member AIAA.

†Professor, Guggenheim School of Aerospace Engineering, Woodruff School of Mechanical Engineering. Associate Fellow AIAA. planned lateral control maneuvers to place the system on final glide at the correct altitude and distance from the target. Human jumpers are able to achieve centimeter-level accuracy using these techniques [11], but performing these lateral maneuvers precisely with an autonomous system is a very challenging problem. The problem is further complicated by deviations from the assumed wind during landing approach, necessitating frequent recalculation of the approach trajectory. Yakimenko et al. showed that deviations in the wind below an altitude of $100 \mathrm{~m}$ can shift the landing point of their system by over $100 \mathrm{~m}$ from the target [10].

The simplest and most direct way of addressing this problem is to provide a means of controlling the glide angle. This allows a guided system to simply point at the target during final approach and adjust the glide angle to compensate for any errors in the approach trajectory or changes in the wind. Slegers et al. demonstrated the use of variable rigging geometry to obtain glide slope control of a parafoil and payload system [12]. For airdrop systems, glide slope is defined as the ratio of the horizontal speed to the vertical speed or, alternatively, the ratio of the ground covered in straight line glide to the initial altitude. Slegers et al. demonstrated controlled glide slope variation from 2.5 to 0.3 for a canopy with an aspect ratio of 2.1 and a variation in glide slope from 3.7 to 2.0 for a canopy with an aspect ratio of 3.8. The current work expands on this initial investigation with flight tests of two high-performance canopies with peak glide ratios of more than 4 . The goals of these tests are to establish the effect of incidence angle on airspeed as well as glide slope and to investigate the coupling of incidence angle and brake deflection as longitudinal control mechanisms.

The paper starts with a discussion of the theory behind variable incidence angle as a glide slope control mechanism. A description is then given of the flight-test hardware, the flight-test methodology, and the steps used in data reduction. Flight-test results exploring the effectiveness of variable incidence angle as a glide slope control mechanism are presented for canopies of two different aspect ratios, flight-test results showing the dynamic response to a change in incidence angle are presented, and the relationship between the incidence angle and symmetric brake and their effects on both the glide slope and airspeed are investigated. Finally, the coupled use of incidence angle and brake deflection to obtain effective glide slope control in different wind conditions is discussed.

\section{Background}

The definitions and sign conventions of canopy incidence angle and symmetric brake deflection used throughout the current work are shown in Fig. 1. A plumb line is drawn from the rigging confluence 


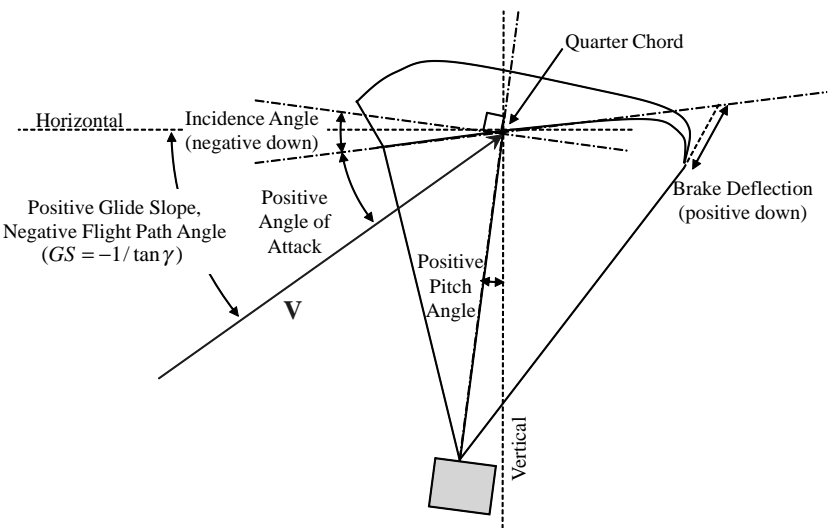

Fig. 1 Sign conventions for incidence angle and brake deflection.

point on the payload through the quarter chord point of the canopy. This line defines the reference frame fixed to the parafoil and payload, and the rotation of this reference frame with respect to the earth is the pitch angle. A chord line is drawn along the bottom of the canopy from the quarter chord to the leading edge. The angle between the horizontal axis of the body fixed reference frame, and this chord line is the canopy incidence angle. A negative incidence angle indicates that the leading edge of the canopy is pulled down from horizontal, and a positive brake deflection indicates that the trailing edge is pulled down from the chord line. This means that a change in incidence angle with constant brake deflection is equivalent to a pure rotation of the canopy.

The system developed by Slegers et al. [12] to demonstrate glide slope control through variable incidence angle is shown in Fig. 2. In addition to the standard control mechanism of symmetric and asymmetric trailing-edge deflection, the canopy rigging can be adjusted in flight to allow longitudinal rotation of the entire canopy about the aerodynamic center to directly control the trim angle of attack in flight.

Flight-test results are shown in Fig. $\underline{3}$, demonstrating that the incidence angle variation can produce a substantial change in the glide angle of the parafoil.

The effect of changing the incidence angle on a parafoil and payload system is very similar to the effect of the elevator on a fixedwing aircraft in glide. Just as the elevator alters the trim angle of attack of the wing, altering the incidence angle produces a change in the trim angle of attack of the parafoil canopy. This means that there is a unique curve of flight-path angle vs angle of attack for a given setting of incidence angle. Similarly, there is a unique lift-to-drag ratio (glide slope) vs angle-of-attack curve determined by the aerodynamic characteristics of a given canopy. The intersection of the flight-path angle curve for a given incidence angle setting and the glide slope

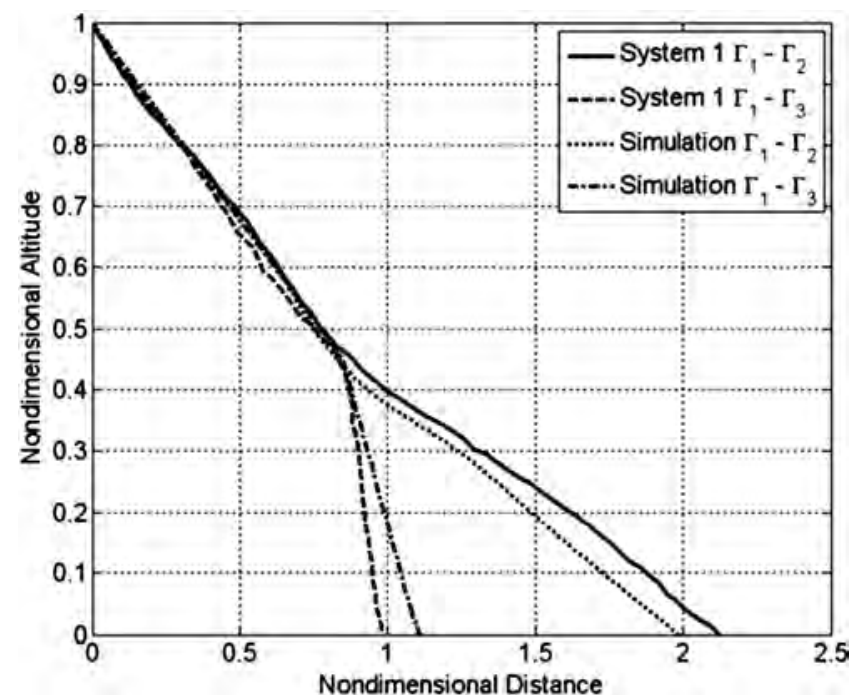

Fig. 3 Flight test results of variable incidence system [12].

curve for a given canopy represents the trimmed flight condition for that combination of incidence angle and canopy. This concept is shown in Fig. 4 for two notional canopies.

The first canopy has a peak glide ratio of 2.5 , whereas the second canopy has a peak glide ratio of 4.5. The important points to note from this plot are that the glide slope range is increased for a more efficient (higher glide ratio) canopy, the sensitivity of glide slope to incidence angle is highest just below the peak glide ratio, and the sensitivity of glide slope to incidence angle is dramatically reduced at low glide ratios. The incidence angle has a more direct influence on flight-path angle than glide slope, and so it is important to keep in mind the nonlinear relationship between glide slope and flight-path angle. A reduction in the glide slope from three to two represents an $8 \mathrm{deg}$ change in the flight-path angle, whereas a reduction in the glide slope from two to one represents an $18 \mathrm{deg}$ change in the flight-path angle. For these reasons, it is more efficient to apply a variable incidence angle as a glide slope control mechanism on canopies with high glide ratios.

Figure 5 shows the glide ratio plotted vs aspect ratio for a variety of parafoil canopies. The data for Snowflake, ALEX, and X-38 canopy performance are published in $[10,13,14]$. The MC-4, Intruder, HiGlide, Firefly, Dragonfly, and Megafly canopy data were obtained from the Airborne Systems' website, and both Skywalk and Gradient paraglider performance data were obtained from the manufacturer's websites. These data are meant to serve only to illustrate the general trend that glide ratio is increased by increasing the canopy aspect ratio. There are two distinct groups in this plot; the lower-aspectratio/lower-glide-ratio group is composed of airdrop systems, and the
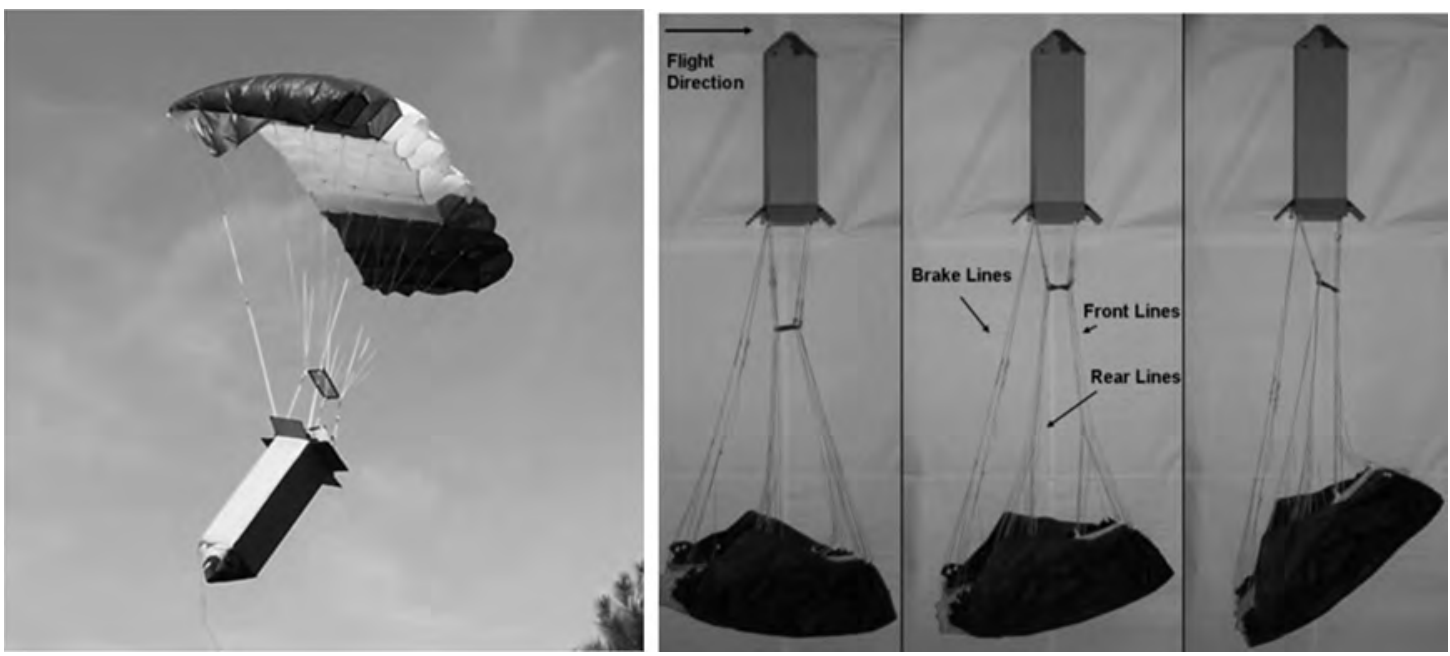

Fig. 2 Variable incidence angle tested by Slegers et al. [12] 

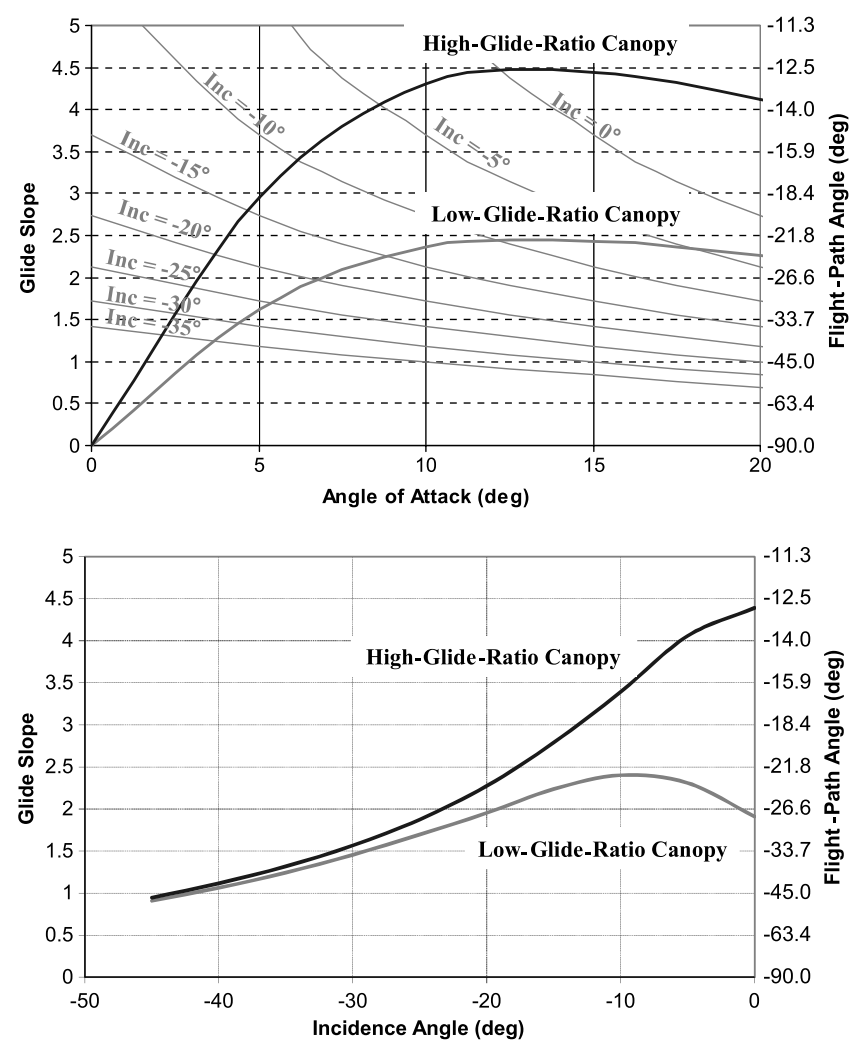

Fig. 4 Conceptual plot of canopy trim conditions.

higher-aspect-ratio/higher-glide-ratio group is composed of paragliding canopies designed for soaring flight. The canopies used for the current work are denoted GT-Imp and GT-Flow. These canopies were originally intended for use as kites. However, in terms of aspect ratio and glide ratio, these canopies lie at the higher end of the performance spectrum of airdrop systems.

\section{Description of Flight-Test Hardware}

An experimental flight-test program was conducted to explore the ability of in-flight incidence angle changes to control glide slope. A self-powered parafoil and payload system was developed to allow rapid flight testing without the use of a drop plane. The system uses an remote control aircraft-style gas motor. Control is achieved with the use of three winch servos. Two winch servos are used to control the left and right brakes independently, and a single winch servo is used to control canopy incidence angle. The payload contains a sensor suite including a GPS receiver and barometric altimeter as well as a flight computer for autonomous control input and data logging. The self-powered payload is shown in Fig. $\underline{6}$.

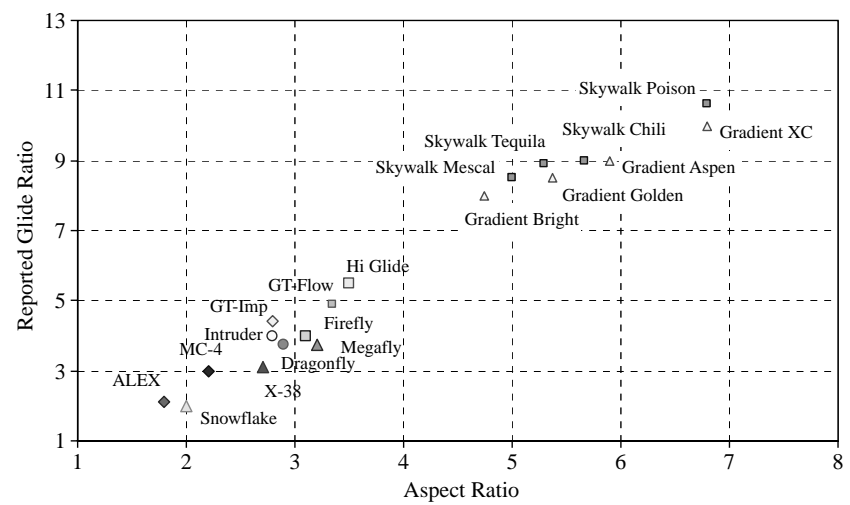

Fig. 5 Reported glide ratio vs aspect ratio for several parafoils.
Two canopies with aspect ratios of approximately 2.8 and 3.4 were tested. The canopy planforms and line attachment points are shown in Figs. 7 and 8. The canopy attachment points were split into four groups. "A" lines all run to the incidence angle control winch, "B" lines and tip lines run to fixed attachment points on the payload, and brake lines run to the left and right brake winches.

By trimming the A lines in concert with the brakes, a pure longitudinal rotation of the canopy to different incidence angles can be achieved, as shown in Fig. 9. This provides direct control over the trim angle of attack, allowing the full range of the canopy's lift-to-drag ratio to be used in flight.

The canopies are designed to be rigged in a style more typical of paragliders than airdrop systems. The center $60 \%$ of the canopy span is a circular arc with a radius equal to $70 \%$ of the canopy span. The outboard sections of the canopy are transitioned from this circular arc to be tangent to the vertical at the wingtips. The low- and mediumaspect-ratio canopies were rigged to generate this same shape. The resulting rigging geometries are shown in Figs. 10 and 11. A photo depicting the actual geometry of the medium-aspect-ratio canopy in flight is shown in Fig. 12, and a comparison of the low- and mediumaspect-ratio canopies in flight is shown in Fig. 13.

The canopy geometry, rigging geometry, and mass properties of the low- and medium-aspect-ratio test systems are given in Table 1. The medium-aspect-ratio canopy is slightly larger than the lowaspect-ratio canopy, and so ballast was added when flying the medium-aspect-ratio canopy to maintain a similar wing loading. The ballast consisted of lead plates mounted to the estimated location of the center of gravity of the payload.

\section{Flight-Test Procedure and Data Reduction}

The flight tests were focused on obtaining steady-state values of airspeed and glide slope as a function of incidence angle and brake deflection. The flight-test procedure begins by climbing under power up to the test altitude. Once the testing altitude is reached, power to the motor is cut, and the incidence angle and symmetric brake level are set to preprogrammed settings. The data logger is switched on and GPS and barometric altimeter data are recorded for approximately $20 \mathrm{~s}$ of gliding flight. The system is then sent back up to the test altitude to repeat the procedure for the next control setting. For each setting of the incidence angle and symmetric brake, a small amount of asymmetric brake is applied to produce a noticeable turn rate (normally $5-15 \mathrm{deg} / \mathrm{s}$ ) to provide sufficient variation in the heading angle to allow a reliable estimate of the wind to be extracted. If a noticeable turn rate could not be achieved with less than $2 \mathrm{~cm}$ of brake differential, then the constant control segment was interrupted after approximately $10 \mathrm{~s}$, the pilot took control and turned the system manually through approximately $180 \mathrm{deg}$, and the constant control segment was continued for roughly another $10 \mathrm{~s}$ on the new heading angle.

Estimates of the atmospheric wind and forward airspeed were generated based on the vector diagram in Fig. 14. The airspeed and wind vector are assumed constant for each segment of the flight in which a constant control deflection is held. The airspeed and wind vector are estimated simultaneously for each constant control segment using an optimizer to minimize the difference between the measured ground track velocity $V_{G}$ and the estimated ground track velocity (computed as the sum of the estimated airspeed and wind vectors).

This process works well when each constant control segment covers a large change in azimuth to expose the wind (e.g., if a control input is held long enough to fly a complete circle, the airspeed is just the average speed measured over the circle and the wind vector is determined from the drift of the circle). The estimation process breaks down if a constant control segment does not contain enough azimuthal variation (e.g., if the vehicle flies in a straight line during the constant control segment, it is impossible to extract separate estimates of the airspeed and wind vector). Each flight normally consists of a series of these constant control segments during which the data are recorded. The segments are performed in quick succession, and so it is reasonable to assume that the wind vectors for 

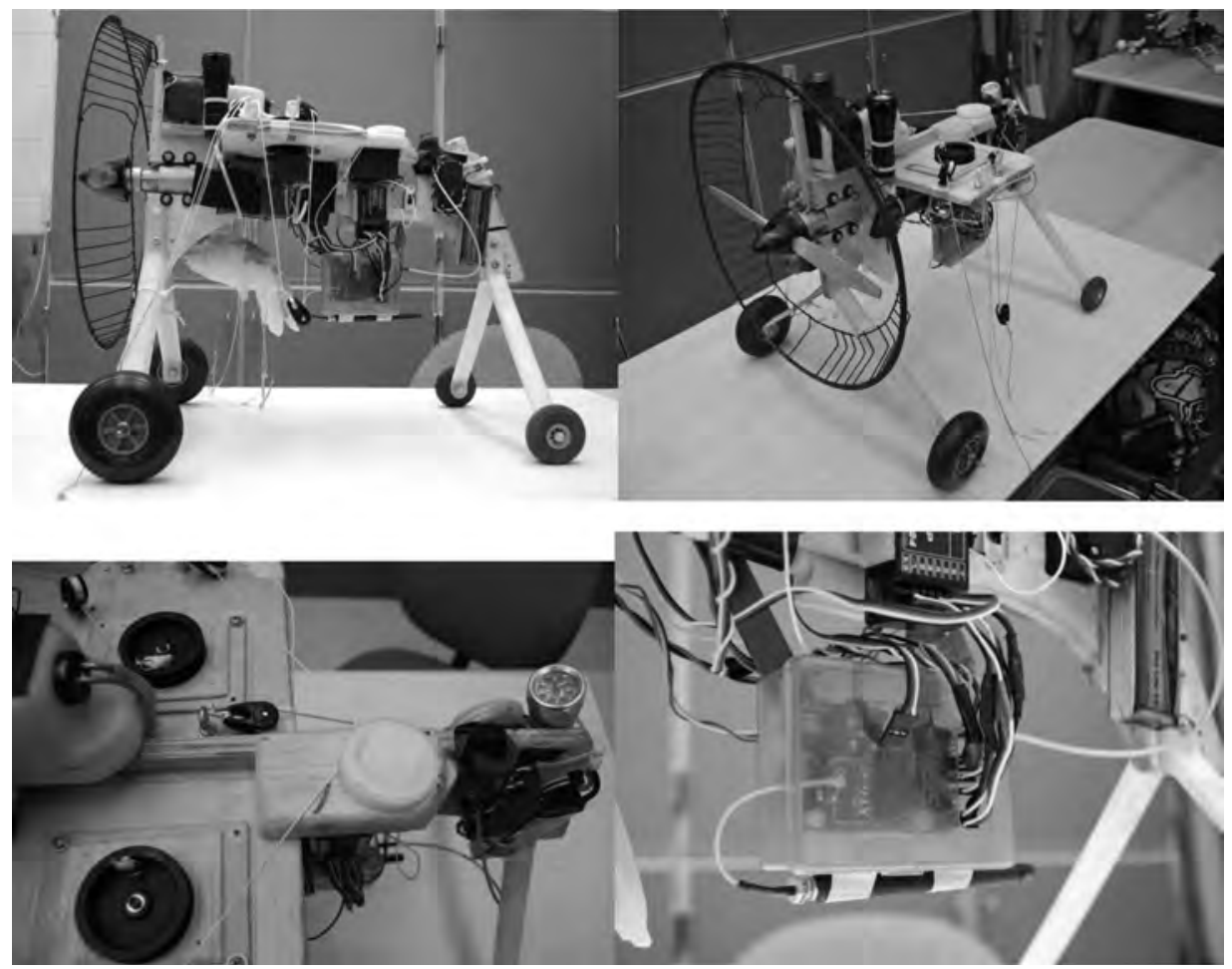

Fig. 6 Flight-test vehicle (bottom left: winch servos, bottom right: flight computer).

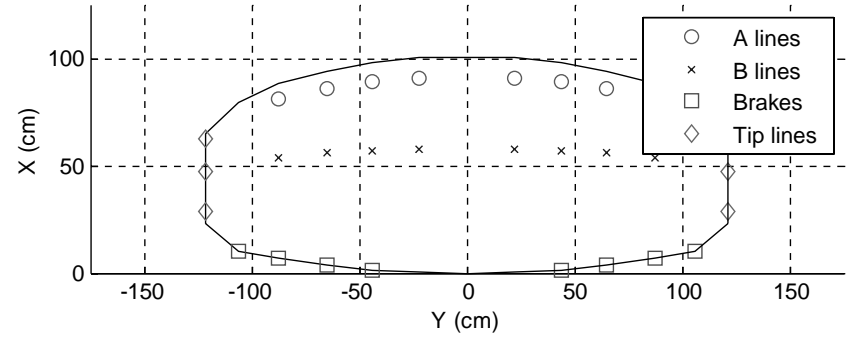

Fig. 7 Low-AR canopy planform showing attachment points.

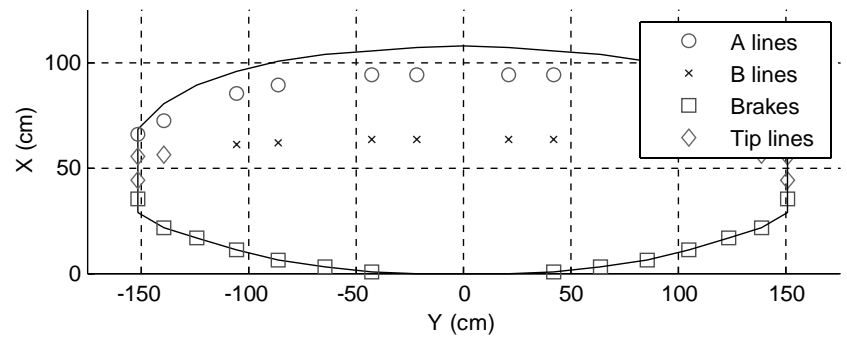

Fig. 8 Medium-AR canopy planform showing attachment points.

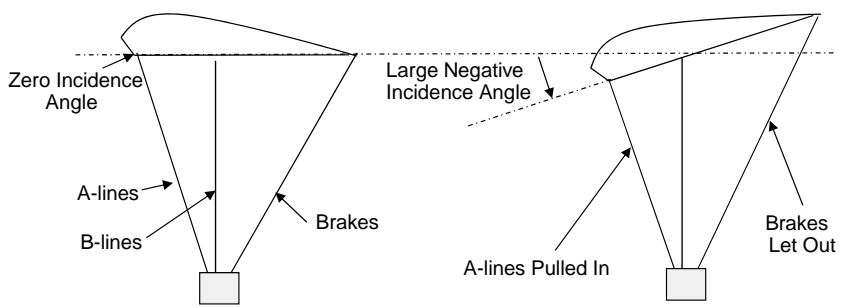

Fig. 9 Incidence angle control.

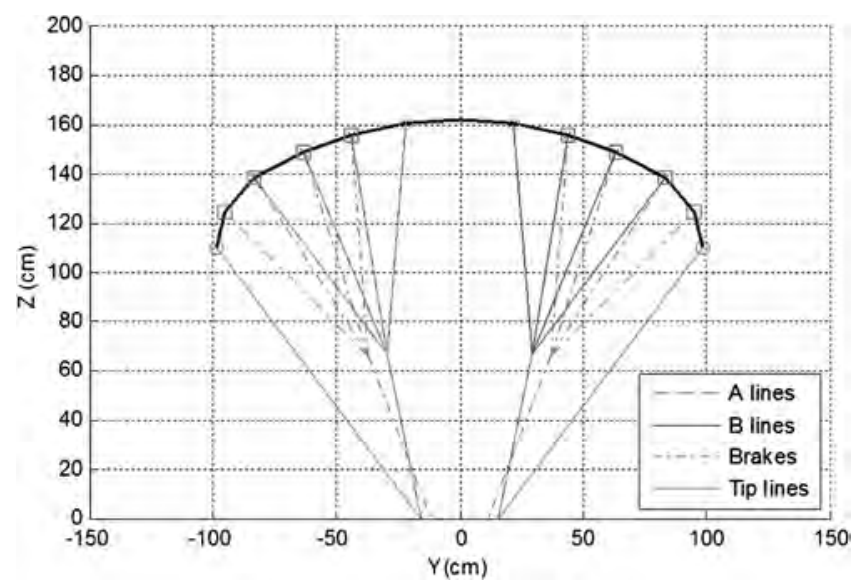

Fig. 10 Low-AR canopy rigging geometry.

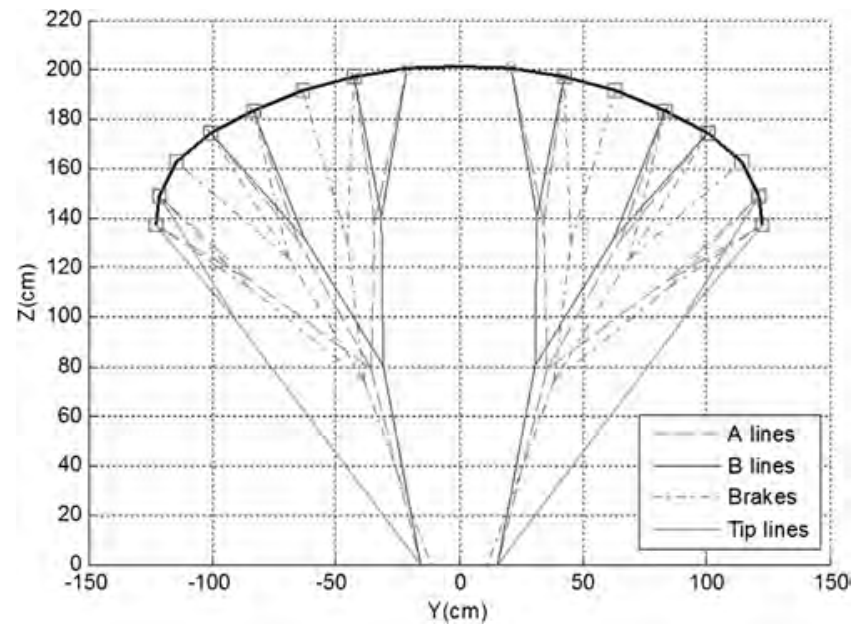

Fig. 11 Medium-AR canopy rigging geometry. 
two segments recorded one right after the other during the same flight should be similar. This assumption is implemented by appending a penalty to the optimization cost function proportional to the difference in the estimated wind vector between successive flight segments during the same flight. This means that if there is no unique solution for the airspeed and wind vector combination from a given flight segment, the optimizer will set the wind vector for this problematic segment to match the wind vectors estimated from the flight segments recorded just before and just after the segment in question.

Figure 15 shows a sample GPS ground track for a constant control flight segment. The turn rate for this flight segment is approximately $12 \mathrm{deg} / \mathrm{s}$, and the drift of the ground track during the circling segment is caused by the wind. Figure 16 shows the measured ground speed, the estimated airspeed, and the ground speed reconstructed from the airspeed and wind estimates. Figure 17 shows the descent rate derived from the barometric altimeter reading during the flight segment. The descent rate estimate is obtained as the median of the measured descent rate.

Each segment of constant control gliding flight results in a single data point of forward speed, descent rate, and turn rate for a particular combination of incidence angle and symmetric brake. These speeds are converted into lift and drag coefficients according to Fig. 18 and Eqs. (1-3):

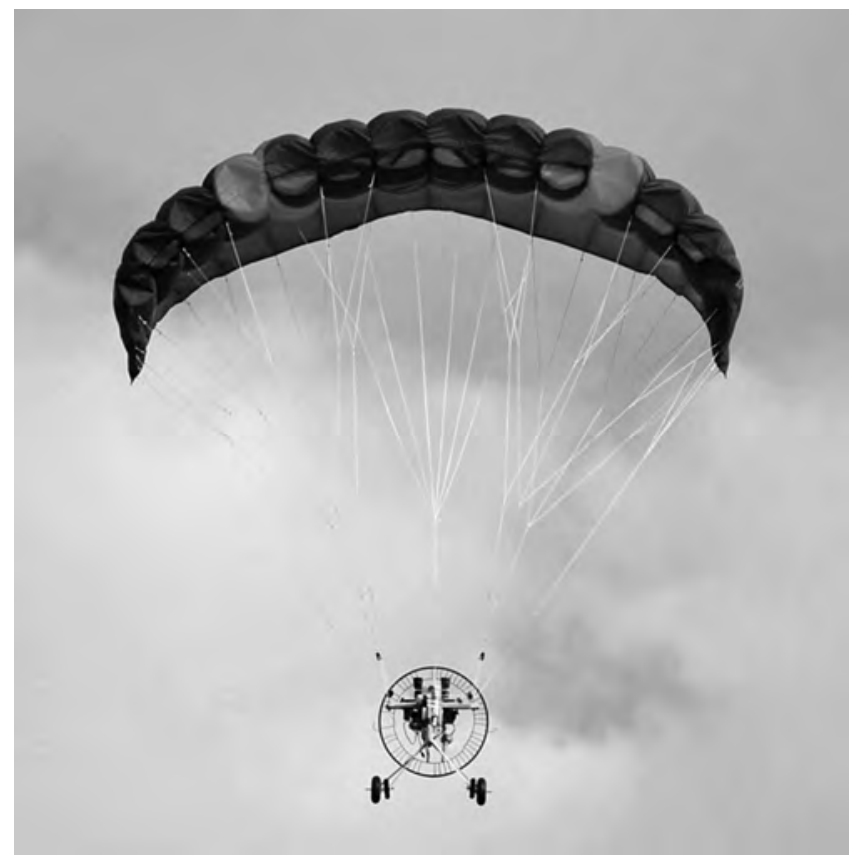

Fig. 12 Medium-aspect-ratio canopy in flight.
Table 1 Canopy, rigging, and payload parameters for flight-test vehicle

\begin{tabular}{lcc}
\hline \hline & Low AR & Medium AR \\
\hline Aspect ratio & 2.79 & 3.35 \\
Area & $2.1 \mathrm{~m}^{2}\left(22.6 \mathrm{ft}^{2}\right)$ & $2.72 \mathrm{~m}^{2}\left(29.3 \mathrm{ft}^{2}\right)$ \\
Span & $2.4 \mathrm{~m}(7.9 \mathrm{ft})$ & $3.0 \mathrm{~m}(9.8 \mathrm{ft})$ \\
Mean chord & $0.88 \mathrm{~m}(2.9 \mathrm{ft})$ & $0.91 \mathrm{~m}(3.0 \mathrm{ft})$ \\
Canopy arc radius & $1.68 \mathrm{~m}(5.5 \mathrm{ft})$ & $2.1 \mathrm{~m}(6.9 \mathrm{ft})$ \\
Projected aspect ratio & 2.01 & 2.39 \\
Projected area & $1.7 \mathrm{~m}^{2}\left(18.3 \mathrm{ft}^{2}\right)$ & $2.23 \mathrm{~m}^{2}\left(24.0 \mathrm{ft}^{2}\right)$ \\
Total rigging line length & $26 \mathrm{~m}(84 \mathrm{ft})$ & $57 \mathrm{~m}(187 \mathrm{ft})$ \\
Mass (weight) & $3.7 \mathrm{~kg}(8.1 \mathrm{lb})$ & $4.72 \mathrm{~kg}(10.4 \mathrm{lb})$ \\
Wing loading & $1.76 \mathrm{~kg} / \mathrm{m}^{2}\left(0.36 \mathrm{lb} / \mathrm{ft}^{2}\right)$ & $1.74 \mathrm{~m}^{2}\left(0.35 \mathrm{lb} / \mathrm{ft}^{2}\right)$ \\
Mass ratio & 1.01 & 0.88 \\
\hline \hline
\end{tabular}

$$
\begin{gathered}
1 / \tan \gamma=V_{0} / \dot{z}=L / D \\
L^{\prime}=W \cos \gamma \\
L=\sqrt{L^{\prime 2}+\left(m V_{0} \omega\right)^{2}}
\end{gathered}
$$

The lift, drag, and glide slope estimates are all determined by taking the average over a set of measurements, and so confidence intervals may be easily calculated to yield an uncertainty estimate for each data point. For a quantity $x$ estimated from a data segment of length $n$, with mean $x_{\text {mean }}$ and sample standard deviation $s$, a conservative estimate of the $95 \%$ confidence interval for the estimated quantity $x$ assuming that the measurements are corrupted by zero-mean, uncorrelated Gaussian noise is obtained according to Eq. (4) [15]:

$$
x=x_{\text {mean }} \pm 2 s / \sqrt{n}
$$

Confidence intervals are obtained in this matter for every estimate of lift, drag, and glide slope individually. Error bars determined from these confidence interval calculations are placed on each data point when the flight-test results are presented next.

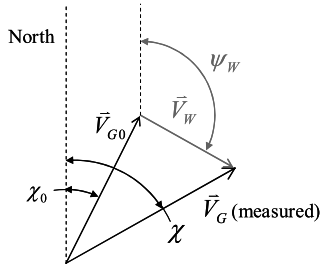

Fig. 14 Decomposition of ground speed vector.

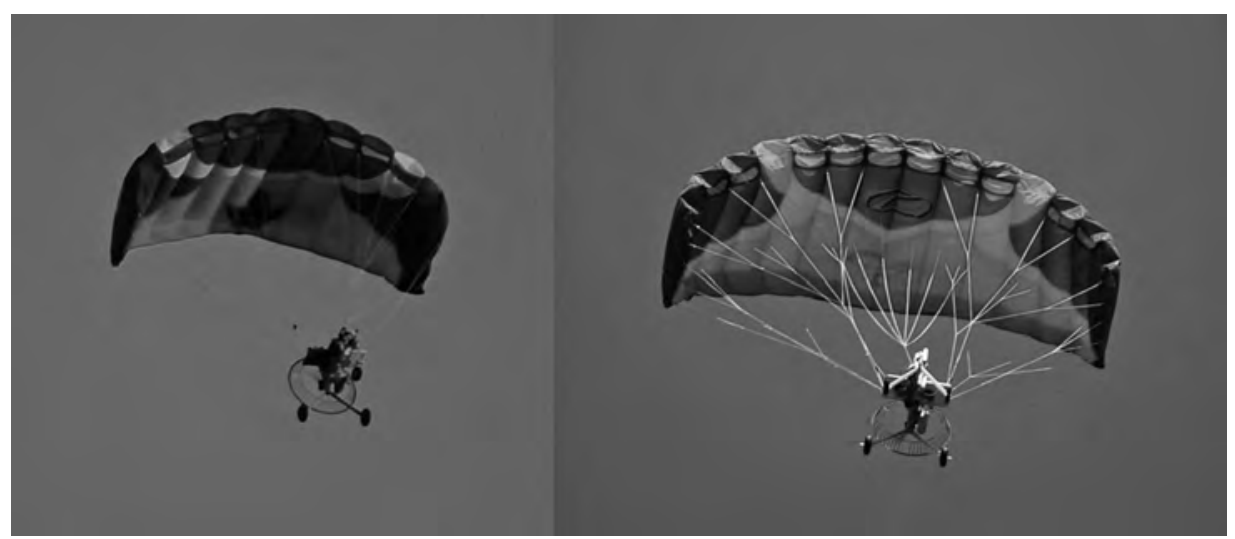

Fig. 13 Comparison of low- and medium-aspect-ratio canopies in flight. 


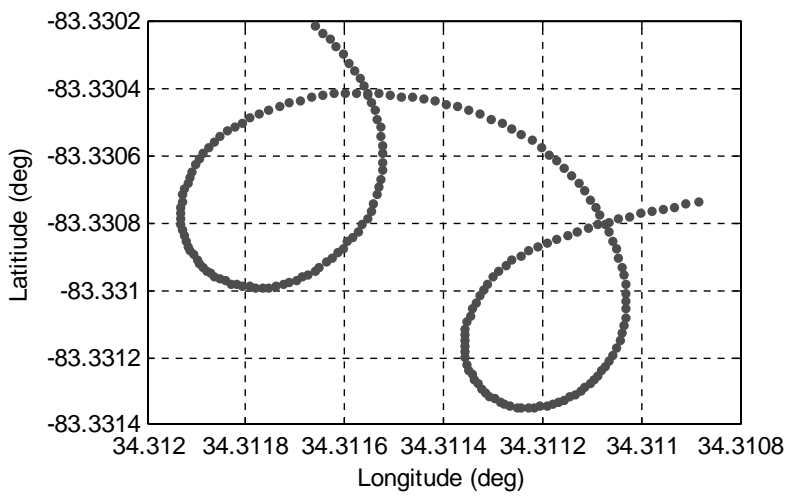

Fig. 15 GPS track for constant control segment.

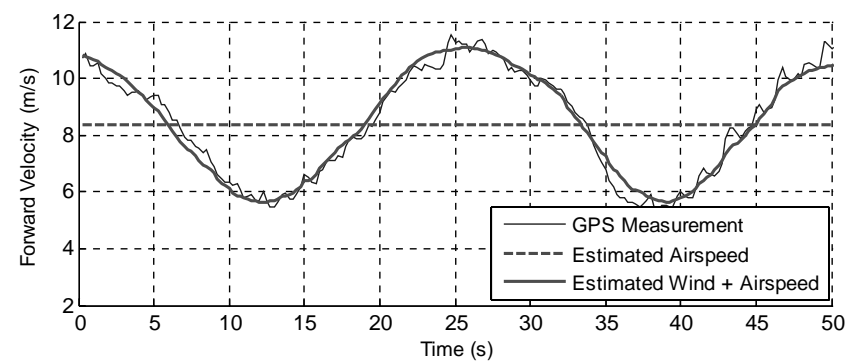

Fig. 16 Extracting forward airspeed from GPS ground speed.

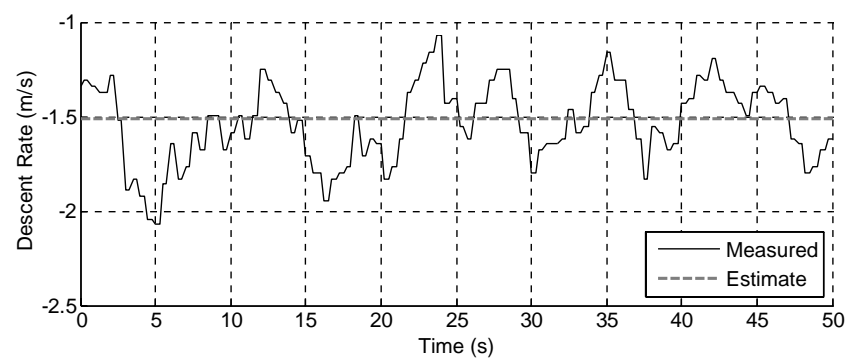

Fig. 17 Descent rate estimate from constant control segment.

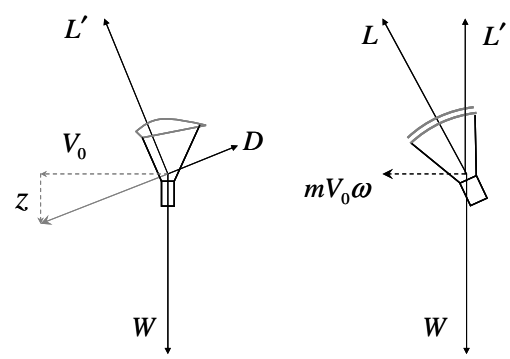

Fig. 18 Estimating lift and drag from forward speed, descent rate, and turn rate.
Table 2 Identified lift and drag parameters with zero brake deflection.

\begin{tabular}{lcc}
\hline \hline Parameter & $\mathrm{AR}=2.8$ & $\mathrm{AR}=3.4$ \\
\hline $\mathrm{C}_{L 0}$ & 0 & 0 \\
$\mathrm{C}_{L A}$ & 3.56 & 4.23 \\
$\mathrm{C}_{L A 3}$ & -28 & -35 \\
$\mathrm{C}_{D 0}$ & 0.075 & 0.102 \\
$\mathrm{C}_{D A 2}$ & 1.072 & 0.310 \\
\hline \hline
\end{tabular}

The variation in the canopy incidence angle produces a change in the angle of attack. The angle of attack can be approximated as the difference between the flight-path angle and the incidence angle. This is an approximation because the pitch angle of the entire system is neglected; however, steady-state pitch angle variations for parafoil and payload systems are normally quite small. The lift and drag coefficients are assumed to vary with angle of attack based on Eqs. (5) and (6). Using the lift, drag, and angle-of-attack estimates extracted from the flight-test data, the aerodynamic parameters in these equations are estimated using linear regression. Finally, the definition of symmetric brake used in the presentation of the results is given in Eq. (7), where $\delta_{R}$ and $\delta_{L}$ are right and left brake deflections, respectively, and $c$ is the mean canopy chord:

$$
C_{L}=C_{L 0}+C_{L A} \alpha+C_{L A 3} \alpha^{3}
$$

$$
C_{D}=C_{D 0}+C_{D A 2} \alpha^{2}
$$

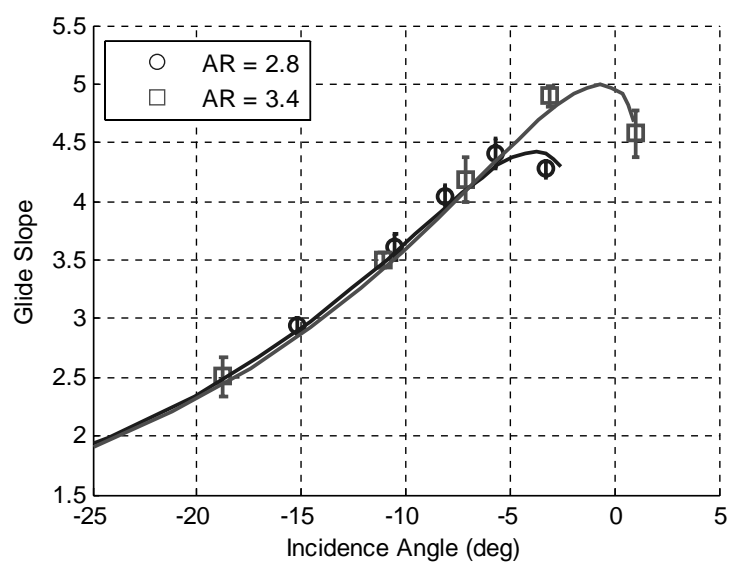

Fig. 20 Glide slope vs incidence angle for low- and medium-aspect-ratio canopies.
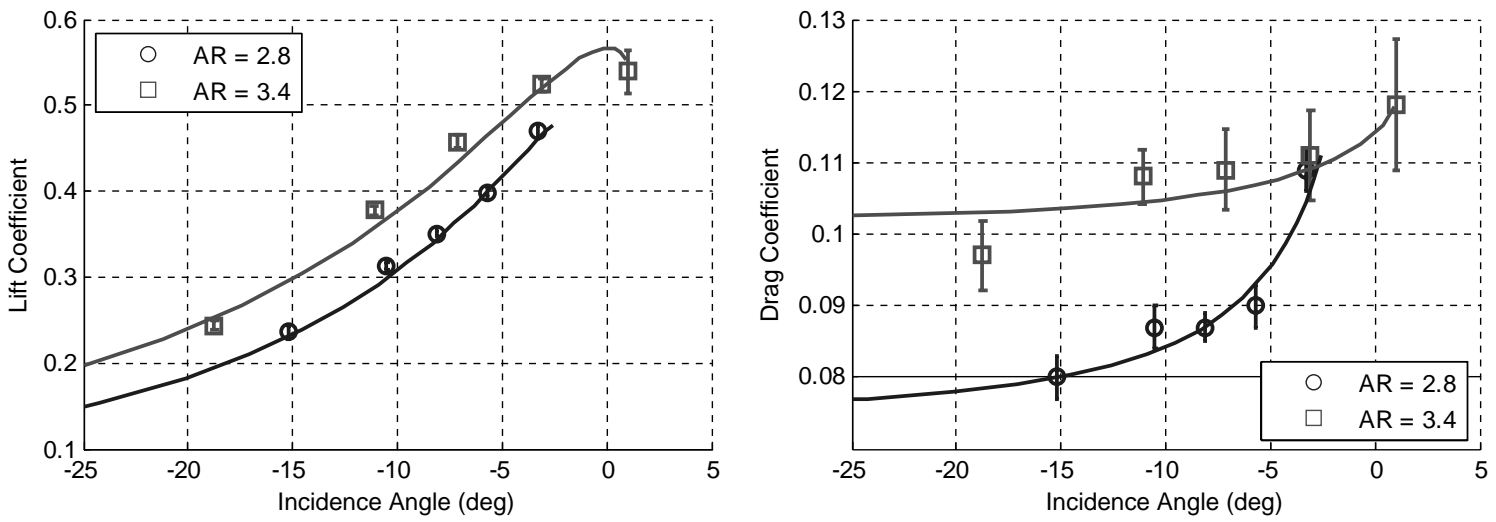

Fig. 19 Lift and drag coefficients vs angle of attack for low- and medium-aspect-ratio canopies. 

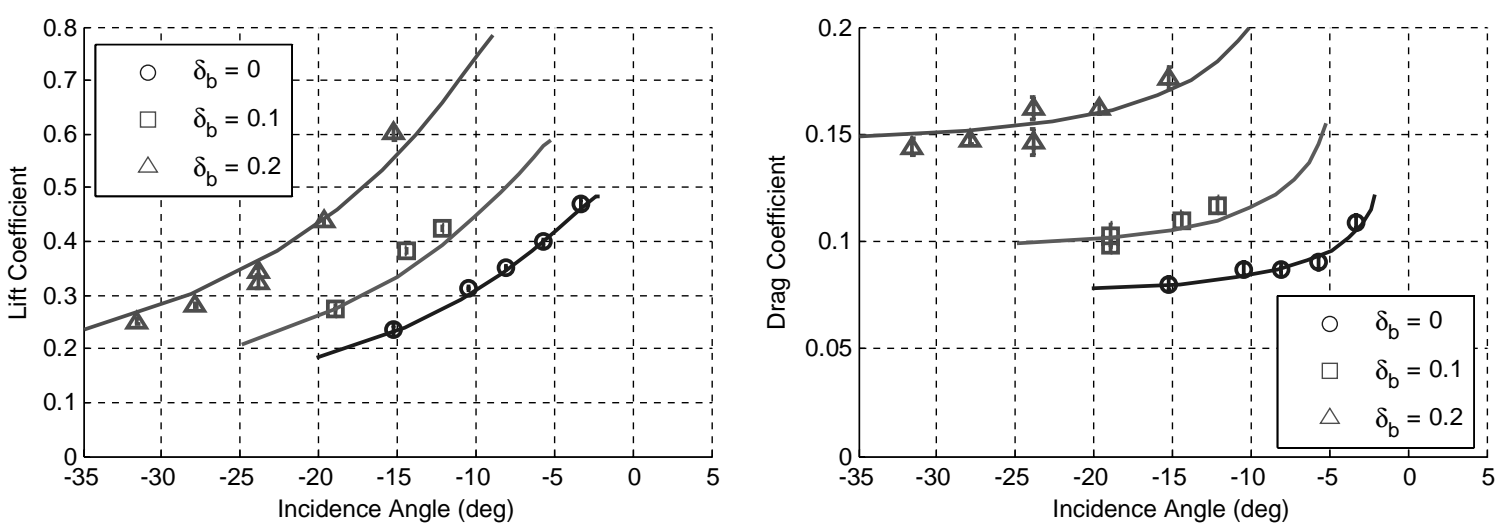

Fig. 21 Lift and drag behaviors vs angle of attack and symmetric brake for low-aspect-ratio canopy.

$$
\delta_{B}=\frac{\left(\delta_{R}+\delta_{L}\right)}{2 c}
$$

\section{Results}

\section{A. Comparison of Low- and Medium-Aspect-Ratio Canopies}

The low- and medium-aspect-ratio canopies were flown at varying incidence angles with zero symmetric brake. In other words, the canopies were rotated through a variety of incidence angles with the brakes trimmed to keep a flat trailing edge. The extracted lift and drag coefficients vs angle-of-attack behaviors for the low- and mediumaspect-ratio canopies are shown in Fig. 19, and the identified aerodynamic parameters are shown in Table 2 . As expected, the lift curve slope for the medium-aspect-ratio canopy is higher than the low-aspect-ratio canopy. However, the medium-aspect-ratio canopy appears to have a higher profile drag coefficient than the low-aspectratio canopy. This may be due to the increased complexity of the rigging for the medium-aspect-ratio canopy, evident in Figs. 10 and 11. Referring to Table 1, the reference area of the medium-aspectratio canopy is only $30 \%$ larger than the low-aspect-ratio canopy, but there is $120 \%$ more rigging line (and line drag) for the mediumaspect-ratio canopy.

The glide slope control achieved by varying incidence angle for these two canopies is shown in Fig. 20. This plot shows that dramatic and effective glide slope control can be achieved by varying the canopy incidence angle. The low-aspect-ratio canopy has a peak glide slope of 4.4, and the medium-aspect-ratio canopy has a peak glide slope of 4.9. The lower limit of glide ratio for the canopies is not well established. There is a minimum angle of attack required to keep the canopies inflated, and so testing near the lower limit of the glide ratio risks a severe frontal collapse of the canopy.

\section{B. Interaction of Incidence Angle and Symmetric Brake}

Flight tests were conducted for the low-aspect-ratio canopy at three levels of symmetric brake. The extracted lift and drag vs angle-ofattack behavior is shown in Fig. 21, and the identified aerodynamic parameters are given in Table 3. The variable incidence provides insight into the effect of symmetric braking that is not normally available from parafoil flight tests. The effect of symmetric brake is typically modeled as producing an increment in both lift and drag.

Table 3 Identified lift and drag characteristics for low-aspect-ratio canopy

\begin{tabular}{lccc}
\hline \hline Parameter & $\delta_{B}=0$ & $\delta_{B}=0.0$ & $\delta_{B}=0.2$ \\
\hline $\mathrm{C}_{L 0}$ & 0 & 0.125 & 0.251 \\
$\mathrm{C}_{L A}$ & 3.56 & 4.06 & 5.06 \\
$\mathrm{C}_{L A 3}$ & -28 & -28 & -28 \\
$\mathrm{C}_{D 0}$ & 0.075 & 0.095 & 0.145 \\
$C_{D A 2}$ & 1.07 & 1.37 & 1.77 \\
\hline \hline
\end{tabular}

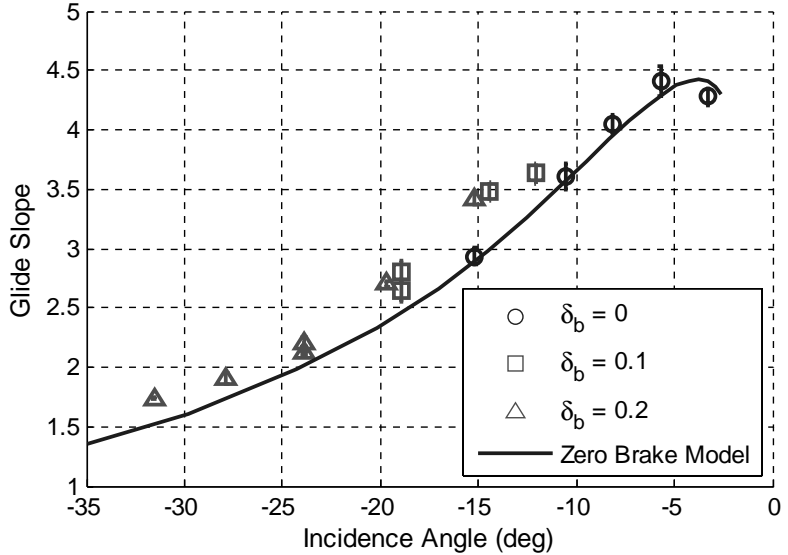

Fig. 22 Glide slope vs incidence for low-aspect-ratio canopy with varying symmetric brake.

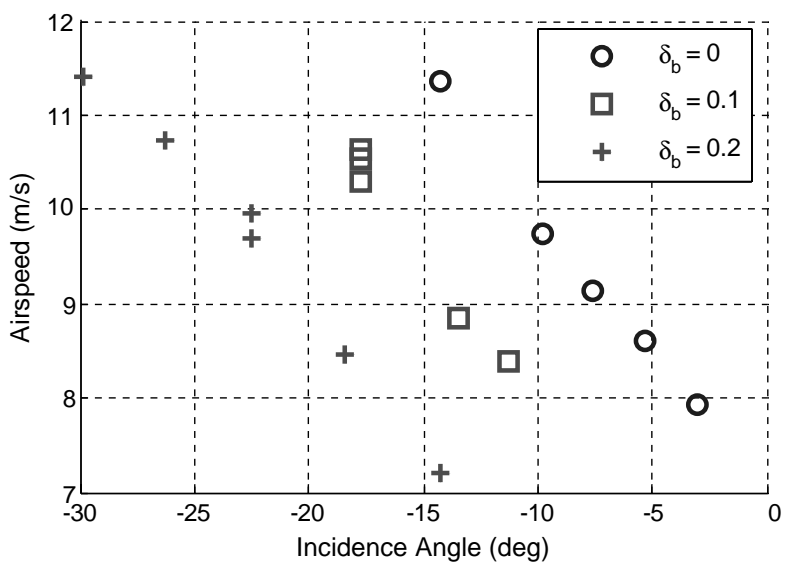

Fig. 23 Airspeed vs incidence angle for low-aspect-ratio canopy.

This effect is evident in the increasing values identified for $C_{L 0}$ and $C_{D 0}$ as brake deflection is increased. However, in addition to this incremental effect, for a given incidence angle the application of symmetric brake also increases the slope of both the lift, $C_{L A}$, and drag, $C_{D A 2}$, curves.

The effect of incidence angle on glide slope at the three symmetric brake levels is shown in Fig. 22. Symmetric braking produces only a modest effect on glide slope. This is consistent with typical airdrop systems in that little change in glide slope is normally achieved with symmetric braking until the system nears stall.

Figure 23 shows the effect of the incidence angle and symmetric brake on airspeed. This plot shows that the incidence angle produces a dramatic effect on the airspeed as well as glide slope. Though symmetric braking is not effective in controlling glide slope, it is quite effective in controlling the airspeed. The relationship between the 


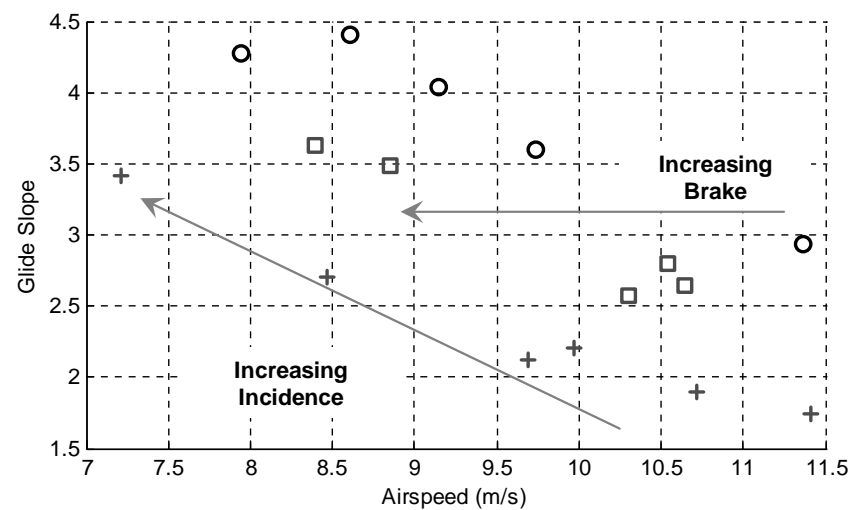

Fig. 24 Glide slope and airspeed envelope for low-aspect-ratio canopy.

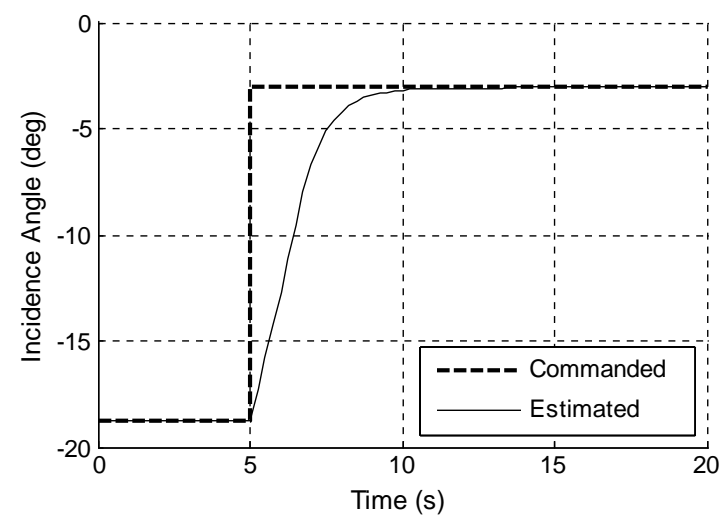

Fig. 25 Dynamic response of medium-aspect-ratio canopy to large increase in incidence angle.

incidence angle and symmetric brake produces the envelope of possible combinations of airspeed and glide slope shown in Fig. 24. This is significant from a guidance and control perspective because it means that the glide slope and airspeed can be controlled independently (within the constraints of the envelope) by modulating the incidence angle and symmetric brake together.

\section{Dynamic Response to Incidence Angle Change}

The dynamic response to a rapid change in incidence angle was recorded for the medium-aspect-ratio canopy. A large increase in incidence angle was commanded with the symmetric brakes held at zero. Figure 25 shows the estimated response of the incidence angle control winch, and Fig. 26 shows the dynamic response of the system to this control input. An oscillation is excited in which the speed and glide ratio are exchanged. Whereas the incidence angle winch servo is able to reach the commanded incidence in roughly $3 \mathrm{~s}$, it takes
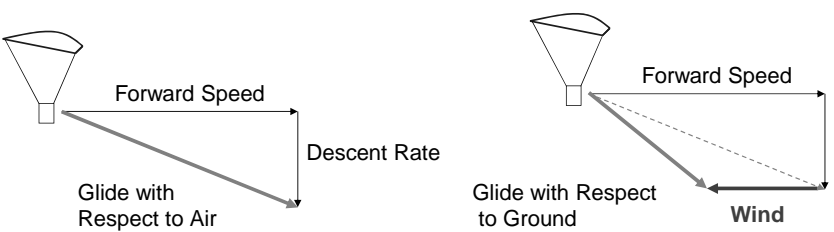

Fig. 27 Glide slope over ground.

approximately $15 \mathrm{~s}$ to reach the new steady-state flight condition. The dynamic response of the low-aspect-ratio canopy was not recorded, but a similar oscillatory response was observed during flight testing.

\section{Control Authority of Glide Slope over Ground}

Variation of the canopy incidence angle can create substantial changes in the glide ratio of a parafoil with respect to the atmosphere. However, it is the glide ratio of the system with respect to the ground that must be controlled to improve landing accuracy. The glide slope over ground is the ratio of the forward speed over ground to the descent rate, in which the forward speed over ground is determined by adding the component of wind aligned with the flight path to the forward airspeed. Figure 27 provides a visualization of the relationship between the aerodynamic glide ratio, wind speed, and glide slope over ground.

This is an important point because, in any amount of wind, the glide slope over ground behaves in a very different manner than the glide slope with respect to the air. The variation in canopy incidence angle is used to vary the angle of attack of the parafoil canopy. The minimum incidence angle results in the minimum angle of attack, which also corresponds to the minimum aerodynamic glide ratio but also the maximum airspeed. As the incidence angle and, hence, angle of attack are increased, the glide ratio is increased, whereas the airspeed is decreased. The consequence of this inverse relationship between the aerodynamic glide angle and airspeed in terms of glide slope over ground is shown conceptually in Fig. 28. In a zero wind environment, the nose-up incidence angle results in an increasing glide ratio over ground. As the wind is increased, the effect of variable incidence angle on glide slope over ground is diminished. In fact, there is a particular wind speed for which the variation in incidence angle will produce no change in the glide slope over ground. Beyond this wind speed, the effect of incidence angle on glide slope over ground is reversed, so that the maximum glide slope over ground is now achieved at the minimum incidence angle setting.

The use of symmetric trailing-edge brake deflection to provide airspeed control in conjunction with variable incidence angle can dramatically improve the range of control of glide slope over ground. Figures 29-32 show the range of glide slope over ground that can be achieved with incidence angle variation alone and with incidence angle variation in conjunction with symmetric brake deflection. These results are based on the flight characteristics of the mediumaspect-ratio canopy used for the variable incidence angle flight tests
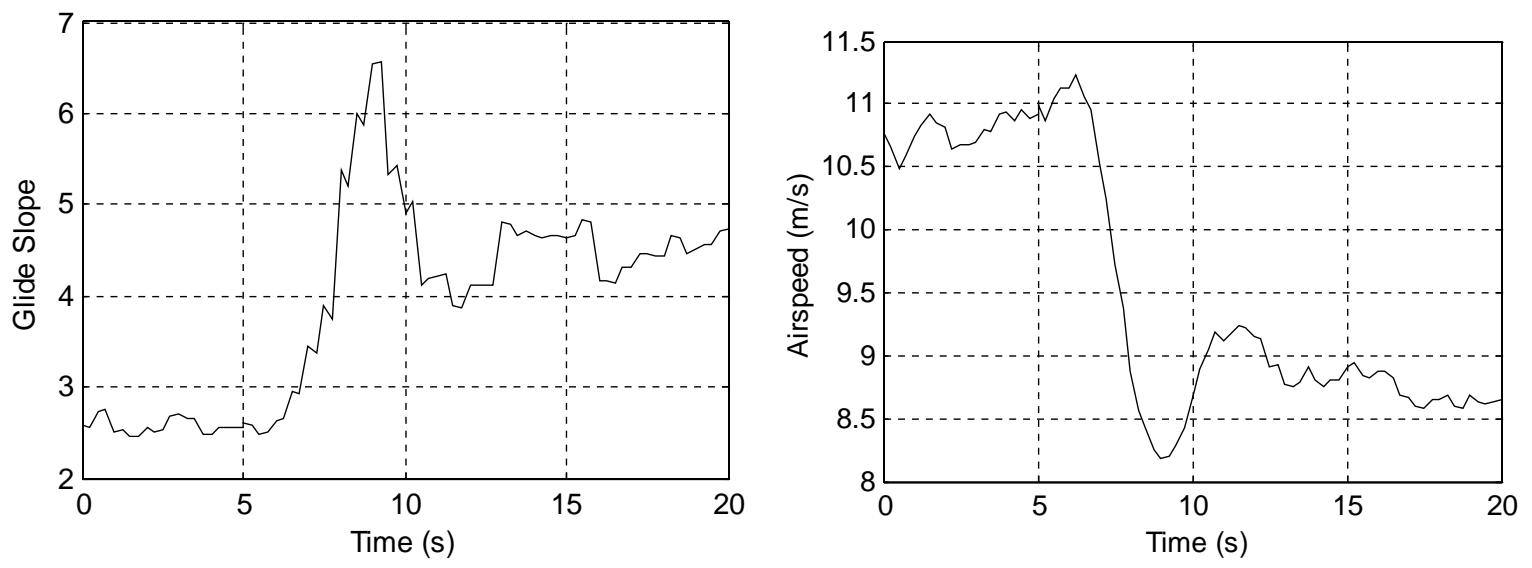

Fig. 26 Dynamic response of medium-aspect-ratio canopy to large increase in incidence angle. 

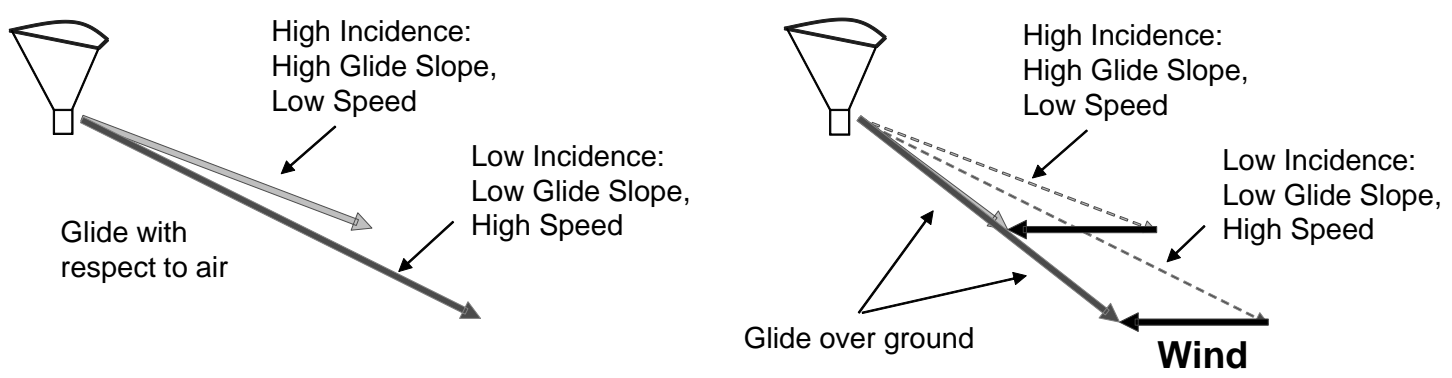

Fig. 28 Behavior of glide slope over ground vs incidence angle.

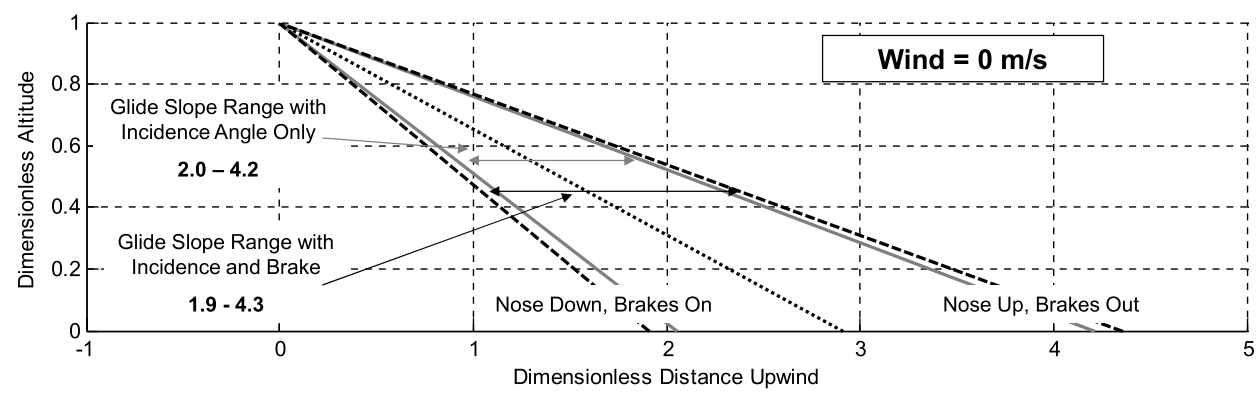

Fig. 29 Range of glide slope over ground in no wind.

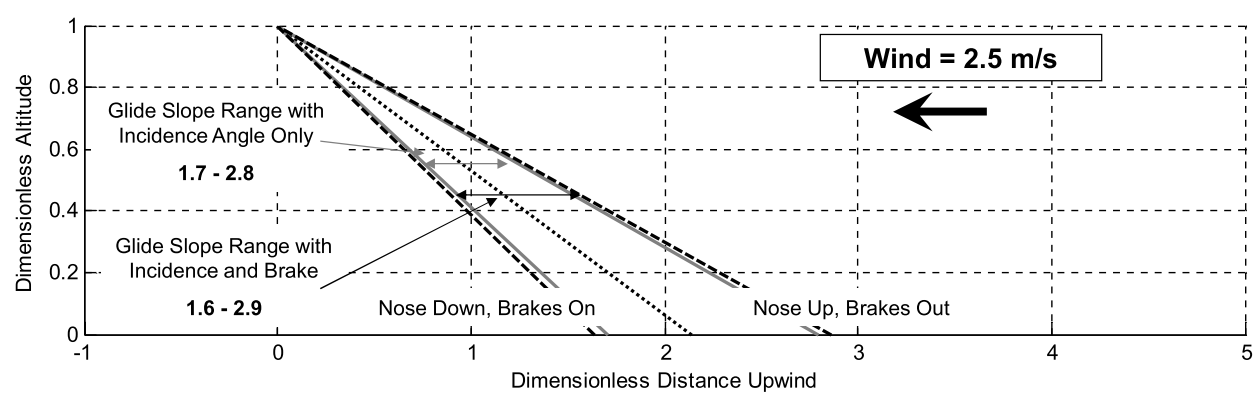

Fig. 30 Range of glide slope over ground in light wind.

discussed in the previous section, but the trends are a direct result of the nature of the incidence angle and symmetric brake control mechanisms. Though the numbers will change, the same behavior is observed with the low-aspect-ratio canopy and, in fact, any other typical parafoil canopy. As shown in Figs. 29 and 30, the variation of the canopy incidence angle provides a significant range of control of glide slope over ground in zero and light wind conditions, whereas the deflection of trailing-edge brakes provides almost no effect on glide slope control over ground. This is because trailing-edge deflection provides a change in airspeed with little change in the aerodynamic glide angle of the parafoil canopy.

However, as shown in Fig. 31, when the wind increases to the point in which variation of the incidence angle produces no change in the glide slope over ground, the use of trailing-edge brake deflection to alter speed can produce a significant range of control in the glide slope over ground. Furthermore, as shown in Fig. 32, the range of glide slope control in stronger wind conditions can be dramatically increased by the use of trailing brakes in conjunction with incidence angle variation.

To implement a coupled incidence angle and symmetric brake controller on an autonomous parafoil and payload system, a model of airspeed and descent rate is developed through the system identification procedure described in this work:

$$
V_{A}=f\left(\Gamma, \delta_{B}\right), \quad \dot{z}=f\left(\Gamma, \delta_{B}\right)
$$

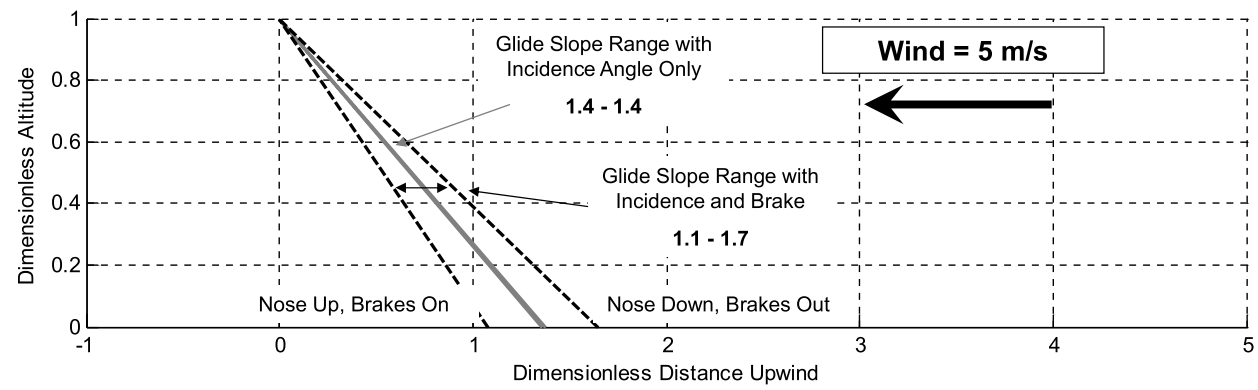

Fig. 31 Range of glide slope over ground in moderate wind. 


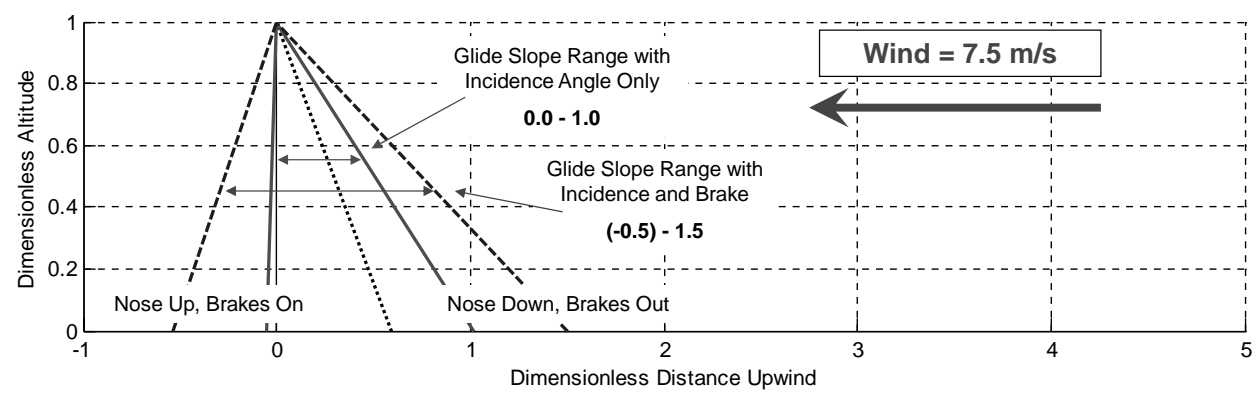

Fig. 32 Range of glide slope over ground in strong wind.

The forward component of speed over ground is determined by subtracting the component of the wind opposing the flight direction from the forward airspeed estimate, and the glide slope over ground is determined as the ratio of the speed over ground to the descent rate:

$$
\mathrm{GS}=\left(V_{A}\left(\Gamma, \delta_{B}\right)-V_{W}\right) / \dot{z}\left(\Gamma, \delta_{B}\right)
$$

Using the models of airspeed and descent rate as functions of the control inputs, a unique mapping can be determined from incidence angle and brake deflection to glide slope over ground for any wind condition. The goal of an autonomous glide slope control algorithm will be to invert this mapping to find the control inputs required to achieve a desired glide slope over ground. It is clear from Eq. (9) that the development of a glide slope control algorithm requires an accurate knowledge of the relationship between airspeed, descent rate, incidence angle, and symmetric brake deflection as well as an accurate estimate of the wind.

\section{Conclusions}

The use of incidence angle as a glide slope control mechanism was explored in theory and tested in experiment. A variable incidence angle system was flight tested with canopies of two different aspect ratios, and dramatic changes in glide slope were demonstrated for both systems. Flight-test results demonstrate that an increase in aspect ratio results in a higher peak glide ratio and extends the controllable range of glide slope. In addition to controlling glide slope, variable incidence angle also produces large changes in airspeed. Because of this coupled effect, the actual change in glide slope over ground achieved by varying the incidence angle can be dramatically reduced in certain wind conditions. The use of trailingedge brake deflection to control airspeed in conjunction with incidence angle variation can compensate for the reduced control authority and ensure effective glide slope control in all wind conditions. A simple method of calculating the relationship between incidence angle, symmetric brake, and glide slope over ground in any wind condition is given to aid in the development of control algorithms using incidence angle variation in conjunction with symmetric braking.

\section{Acknowledgments}

The authors would like to acknowledge the support of the Natick Soldier Research Development and Engineering Center Airdrop Technology Team.

\section{References}

[1] Calise, A., and Preston, D., "Swarming/Flocking and Collision Avoidance for Mass Airdrop of Autonomous Guided Parafoils," Journal of Guidance, Control, and Dynamics, Vol. 31, No. 4, 2008, pp. 1123 1132 doi: $10.2514 / 1.28586$
[2] Carter, D., George, S., Hattis, P., Singh, L., and Tavan, S., "Autonomous Guidance, Navigation, and Control of Large Parafoils," 18th AIAA Aerodynamic Decelerator Systems Technology Conference and Seminar, AIAA Paper 2005-1643, May 2005.

[3] Carter, D., George, S., Hattis, P., McConley, M., Rasmussen, S., Singh, L., and Tavan, S., "Autonomous Large Parafoil Guidance, Navigation, and Control System Design Status," 19th AIAA Aerodynamic Decelerator Systems Technology Conference and Seminar, AIAA Paper 2007-2514, May 2007.

[4] Carter, D., Singh, L., Wholey, L., Rasmussen, S., Barrows, T., George, S., McConley, M., Gibson, C., Tavan, S., and Bagdonovich, B., "BandLimited Guidance and Control of Large Parafoils," 20th AIAA Aerodynamic Decelerator Systems Technology Conference and Seminar, AIAA Paper 2009-2981, May 2009.

[5] Jann, T., "Advanced Features for Autonomous Parafoil Guidance, Navigation and Control," 18th AIAA Aerodynamic Decelerator Systems Technology Conference and Seminar, AIAA Paper 2005-1642, May 2005.

[6] Kaminer, I., and Yakimenko, O., "Development of Control Algorithm for the Autonomous Gliding Delivery System," 17th AIAA Aerodynamic Decelerator Systems Technology Conference and Seminar, AIAA Paper 2003-2116, May 2003.

[7] Murray, J., Sim, A., Neufeld, D., Rennich, P., Norris, S., and Hughes, W., "Further Development and Flight Test of an Autonomous Precision Landing System Using a Parafoil,” NASA TM-4599, July 1994.

[8] Slegers, N., and Yakimenko, O., "Terminal Guidance of Autonomous Parafoils in High Wind to Airspeed Ratios," Journal of Aerospace Engineering, Vol. 225, No. 3, March 2011, pp. 336-346.

[9] Calise, A., and Preston, D., "Design of a Stability Augmentation System for Airdrop of Autonomous Guided Parafoils," AIAA Guidance, Navigation, and Control Conference and Exhibit, AIAA Paper 20066776, Aug. 2006.

[10] Yakimenko, O., Slegers, N., and Tiaden, R., "Development and Testing of the Miniature Aerial Delivery System Snowflake," 20th AIAA Aerodynamic Decelerator Systems Technology Conference and Seminar, AIAA Paper 2009-2980, May 2009.

[11] Bergeron, K., Fejzic, A., and Tavan, S., "AccuGlide 100: Precision Airdrop Guidance and Control via Glide Slope Control," 21st AIAA Aerodynamic Decelerator Systems Technology Conference and Seminar, AIAA Paper 2011-2530, May 2011

[12] Slegers, N., Beyers, E., and Costello, M., "Use of Variable Incidence Angle for Glide Slope Control of Autonomous Parafoils," Journal of Guidance, Control, and Dynamics, Vol. 31, No. 3, 2008, pp. 585-596. doi:10.2514/1.32099

[13] Carter, D., George, S., Hattis, P., McConley, M., Rasmussen, S., Singh, L., and Tavan, S., "Autonomous Large Parafoil Guidance, Navigation, and Control System Design Status," AIAA Paper 2007-2514, May 2007.

[14] Jann, T., "Aerodynamic Model Identification and GNC Design for the Parafoil-Load System ALEX," 16th AIAA Aerodynamic Decelerator Systems Technology Conference and Seminar, AIAA Paper 2001-2015, May 2001.

[15] Klein, V., and Morelli, E., Aircraft System Identification: Theory and Practice, AIAA Education Series, AIAA, Reston, VA, 2006, pp. 103104. 